Received: July 29, 2017

Revision received: June 2, 2018

\title{
Application of Brain Science in Physical Education and Teaching
}

\author{
Jiayi Zhao $^{1}$ \\ North Sichuan Medical College
}

\author{
Guangxue $\mathrm{Li}^{2}$ \\ China West Normal University
}

\begin{abstract}
With the development of science and technology, brain science has obtained fruitful results, which have been widely applied in many fields. For physical education (PE), it is also of great significance to apply the results of brain science research. In order to optimize physical education and teaching methods, this paper explores the possibility of combining brain science with physical education, and studies the application of brain science in physical education and teaching. It then proposes PE teaching arrangements and methods according to the latest brain science research results such as the whole brain theory and the imagery thinking theory. The research results of this paper provide the basis for scientifically formulating the content and objectives of the PE curriculum, and also can promote the scientific and standardized development of physical education and teaching.
\end{abstract}

\section{Keywords}

Brain Science • Physical Education • Teaching Method

\footnotetext{
${ }^{1}$ Jiayi Zhao, North Sichuan Medical Collece, Nanchong 63700, China. Email: 334650499@qq.com

${ }^{2}$ Correspondence to: Guangxue, Li, China West Normal University, Nanchong 63700, China. Email: 334650499@qq.com
} 
In recent years, the research achievements and development prospects of brain science have received extensive attention. The research results have been widely used in various fields, and proved effective in helping develop brain potential and creativity. Brain science also plays an important role in the field of education. On the one hand, it is used to evaluate the scientificity of various quality education practices, and on the other hand, it can also be used to develop excellent teaching methods. Therefore, it is of great significance to establish a relationship between brain science and physical education to optimize this curriculum.

Sports can excavate brain potential, promote intellectual development and enhance brain's cognitive ability and plasticity. On the one hand, physical exercise can improve the function of the central nervous system, promote the development of human observation and perception and increase the coordination and flexibility of the brain; on the other hand, strengthening physical exercise can also enhance the alternation of cerebral cortex inhibition and excitement and improve the accuracy and balance of the cerebral cortical nervous system, which help tap brain potential and promote intellectual development (Saxena \& Flora, 2012; Wi-Young So, 2017). There is no doubt that physical exercise can do good to brain development; however, how to apply brain science in the PE teaching process has not been fully studied, except for some basic research. In addition, as an emerging field, brain science is not well known by parents and PE teachers. Therefore, it is urgent to do more research on brain science and promote its application in PE teaching.

Aiming to optimize physical education and teaching methods, this paper explores the value of brain science research results in the process of physical education teaching, and applies the brain science theory in PE teaching design. First, it analyzes the complex relationship between brain science and physical education, and discusses the effects of physical sports on human brain's cognitive ability and plasticity. Secondly, this paper explores how to apply the relevant theories and research results of brain science to the PE teaching process and puts forward PE teaching arrangements and new teaching methods to improve the quality of physical education. The research results of this paper provide scientific theoretical support for high quality PE teaching, and give a guidance on how to improve the quality of physical sports.

The rest of the paper is organized as follows. Section 2 discusses brain science and PE teaching, with a focus on the close relationship between brain science and physical education; Section 3 studies the application of brain science in physical education, and puts forward PE teaching arrangements and new teaching methods; the paper is concluded in Section 5.

\section{Brain Science and Physical and Sports Education}

\section{Overview of brain science}

The theories of brain science describe how the common activities of neuronal cells in the brain generate behaviours and analyze why the brain activity is simulated or affected by the environment, and then theoretically describe the advanced cognitive behaviours of the brain, including language, intelligence, thinking and perception, etc. (Gu et al., 2015). In the broad sense, brain science studies human brain structure and functions at the cellular, molecular and psychological levels, and expounds the close interdisciplinary relationships 
between the higher consciousness function of the human brain and education and cognitive science. Brain is the most complex structure in the human body. Revealing how the brain works has become the biggest challenge in modern brain science. The cerebral cortex is the centre of the brain's advanced functions, which is divided into four regions, namely the parietal lobe, occipital lobe, temporal lobe and frontal lobe. Although they have their respective tasks, studies have shown that many advanced functions require the participation of different regions (Foster, Lyte, Meyer \& Cryan, 2016). The practice of brain science reveals that the nervous system of the brain is highly plastic, and for individuals, different functional regions have their own critical development periods. Under the stimulation of the external environment, the nervous system of the brain can continuously train and strengthen its own structure and functions (Yang et al., 2016). So far, brain science has achieved some results in the brain cognitive ability development (Fjell et al., 2015), brain functional localization (Yang et al., 2014), whole brain theory and imagery thinking theory. Since thinking model and cognitive ability are the core of brain science education, the cognitive learning and training based on brain science has now become a new direction for educators.

\section{Physical exercise enhances brain cognition}

Brain cognition is an intelligent process in which the body recognizes and acquires knowledge. This process involves a series of social and psychological behaviours such as spirit, emotion, learning and thinking. Sports can not only strengthen the body, but also promote the growth of nerve cells and enhance the brain's cognitive ability, including memory, attention and mental health. First of all, in terms of memory, experiments conducted among people at different ages show that physical health would make the brain structure hippocampus grow well. In the brain structure, the hippocampus is essential to memory and brain learning, and it responds strongly to aerobic exercise, suggesting that increasing exercise can not only slowly improve memory, but also rapidly affect memory formation. On the other hand, sports and exercise can also help boost your brain's attention. In a test on German students, half an hour of aerobic exercise boosted the attention of these students. Through physical exercise, students can better control and manage information in their brains, avoid distractions, and perform multiple tasks simultaneously. In addition, physical exercise can also affect the mood of the human brain and further improve mental health. After finishing running or other sports, people will feel more comfortable. This excitement stems from the endogenous cannabinoid system.

Regular exercise can improve people's cognitive performance, resist age-related cognitive decline and enhance people's emotions. On the one hand, physical exercise can improve the function of the central nervous system, promote the development of human observation and perception and increase the coordination and flexibility of the brain; on the other hand, strengthening physical exercise can also enhance the alternation of cerebral cortex inhibition and excitement and improve the accuracy and balance of the cerebral cortical nervous system, which help tap brain potential and promote intellectual development. As exercise increases and continues, personality characteristics will show externally, and tension and anxiety will decrease. What is more, the individual's competitiveness and creativity will be strengthened, and overall self-control and persistence improved. These ability enhancements will give the individual more energy to learn.

Different sports such as mind-body exercise, aerobic exercise, resistance exercise and sensitive exercise 
have different effects on the cognitive function. Specifically, resistance exercise and strength exercises can effectively delay the degradation of strength, increase explosive power, and improve the cognitive function. Mind-body exercise not only improves the state of mind, but also changes the structure of the central nervous system and makes the cerebral cortex thicker. Exercise frequency and intensity also have certain impacts on the cognitive function. The physical activity is positively correlated with the cognitive function, which means there is a dose-effect relationship between the two. The changes in various factors like frequency, intensity and time and their interactions improve the cognitive function. According to an investigation, physical exercise has a significant impact on the cognitive level of the elderly. The more frequently the elderly take exercise every week, the higher their cognitive level will be, and what is more, group exercise can contribute more to the improvement of the cognitive ability (Li, Dai \& Chen, 2012).

\section{Physical exercise improves brain plasticity}

The brain is a complex dynamic system whose function and structure are gradually formed during the growth and development process. Under the influence of factors such as training, learning and experience, the cerebral cortex will experience certain functional reorganization and structural changes during the development process, making the brain plastic. In the whole process of human growth, the central nervous system has always been plastic to a certain degree, and it is a lifelong ability. Such plasticity refers to the ability of the brain to constantly adjust and reorganize its functions and structure as the internal and external environment changes. This ability makes the central nervous system highly adaptable to different environments. In terms of human health, a very important goal is to maintain the brain's good plasticity, so that the brain can always be in normal operation. Despite many ways to improve brain plasticity, physical exercise remains to be a very efficient and feasible method. Therefore, it is important to study the effects of physical exercise on the plasticity of the brain and fully understand the relationship between the two.

The results of brain cognitive science research show that regular aerobic exercise can promote the blood supply of the heart to the brain and the body, so that the blood will contain more oxygen and make the brain cells healthier. Since the brain is plastic, physical exercise will play an important part in improving its perception, memory and thinking. At the same time, some studies have also proved that appropriate sports activities can help build individuals' full personalities. In the clinical rehabilitation of patients with brain injuries, intensive exercise skill practice, rich environment stimulation and aerobic exercise are proved useful in promoting brain plasticity. The reasons why the physical exercise works are that it promotes the expansion of blood vessels in the brain, increases blood flow and hormones and helps generate more new nerve cells. Therefore, from the perspective of brain science, students need to carry out physical activities more frequently during their growth to release the potentials of their brains.

The impacts of physical exercise on the plasticity of the brain are mainly manifested in the macroscopic, cellular and molecular levels. On the macro level, physical exercise can promote brain plasticity mainly in regions like hippocampus, cerebellum and dorsal striatum. Moderate exercise can slow down the decline of white matter and gray matter in the temporal, frontal and parietal lobes. On the cellular level, the benefit of physical exercise is mainly manifested in neurogenesis. Neural stem cells can produce new neurons under 
certain inducing factors. On the molecular level, physical exercise can promote the production and secretion of growth factors such as insulin-like growth factor and neuropeptide in the human body (Vaynman, Ying \& Gomez-Pinilla, 2004).

\section{Application of brain science in PE teaching}

In recent years, more and more brain science research results have brought new inspirations to the content and teaching methods of different curriculums, particularly PE. This section applies relevant theoretical results of brain science to PE teaching and proposes teaching arrangements and new methods to improve the teaching quality of the curriculum.

\section{Content arrangement of PE teaching}

At present, PE teaching has a high degree of freedom. Teachers can arrange the schedules according to their own abilities and choose the PE content at their own discretion; and on the other hand, students can choose sports according to their preferences. However, under this model, it is difficult to cultivate all-round talents. Sports can not only enhance physical fitness, but also help develop students' emotional, cognitive, attention and immune systems and other neurobiological system. Therefore, it is very necessary to arrange the teaching course and content according to the specific physical exercise needs of different students to develop them in an allround way.

Sports can promote the development of the cognitive system, and at the same time it also involves the performance and participation of the executive function. As the core of the cognitive system, the executive function refers to the high-level cognitive process involved when the complex cognitive task is being completed. In real life, students always have some problems with their executive functions, so the content of PE can be set according to the effects of sports on the executive function. Specifically, the analysis can be carried out from three aspects - environment, techniques and tactics and intensity. For example, the three-on-three half-court basketball game focuses on cultivating and improving students' offence-defence conversion, while exercise, martial arts and aerobics focus on cultivating and improving their refreshing functions. PE teachers need to set sports teaching content according to the different needs of the students, so as to improve their executive functions.

Appropriate sports can improve students' mood and ease their stress, so it is necessary to arrange appropriate PE content. As a complex reaction of human beings, emotions mainly involve psychological state, physiological change and reaction impulse. Stress is an important part of emotions and also an important condition based on which humans adapt to different complex environments. Appropriate amount of stress can continuously promote students' learning and cognitive abilities. Experimental studies have shown that sports have positive psychological effects on students, and that different sports have different effects (Li \& Wang, 2012). For example, basketball, tennis, martial arts and aerobics can stimulate more vitality, so that students can get the psychological pleasure of sports. Therefore, PE teachers should arrange the PE courses at different intensities, time and frequencies according to students' needs to adjust their mood or relieve their stress. 


\section{New PE teaching methods}

The PE curriculum must be implemented according to scientific programs and methods. This section applies the theoretical results of brain science to the PE teaching process. By analyzing the latest brain science research results such as the whole brain theory and the imagery thinking theory, it proposes new appropriate PE teaching methods.

According to the whole brain theory, human brain thinking can be divided into four types - organizational thinking, communicative thinking, analytical thinking and fantasy thinking, which are distributed in different regions of the brain. The organizational and analytical thinking is controlled by the left hemisphere of the brain, while the communicative and fantasy thinking by the right hemisphere (Herrmann, 1997). The brain is an integral organism, where the above four types of thinking must be connected and utilized together. However, in actual physical education, students generally exercise only one side of their bodies, and teachers pay little attention to developing both sides, which is not very helpful to developing their thinking abilities. Therefore, in the process of PE teaching, teachers should consciously strengthen the sports training of the weaker limbs of the students, and adjust the content of physical education so that students can have more balanced development.

According to the theory of imagery thinking, the processes of representation, perception and motion are controlled by a common brain mechanism. The motion processing simulated in the brain is consistent with that of the real object (Zhang, 2004). Physical education is a process involving the demonstration by teachers and the imitation by students, in which, it is very important to present the correct movements and help students develop visual images. The use of multimedia applications such as slides and videos in physical education can break the limitations of the demonstrations made by teachers. In the multimedia PE teaching process, teachers can emphasize the technical aspects and details of each movement so as to enhance students' intuitive vision and provide clearer visual representations for them. Teachers should encourage students to learn through instructional videos after class to strengthen their representation training and accelerate the improvement of their motor skills.

\section{Conclusion}

The results of brain science research have been fruitful and widely used in many fields. When applied in PE teaching, they are also very helpful, as brain science has a close relationship with physical education. The results of brain science research can help develop brain potential and creativity efficiently and achieve scientific sports training. This paper focuses on studying the physical education and teaching methods based on brain science. Firstly, it analyzes the close relationship between brain science and physical education, and then proposes the PE content arrangements and new PE teaching methods based on the latest brain research results such as the whole brain theory and the imagery thinking theory. The research results of this paper can be applied in PE classroom teaching and provide practical teaching methods and scientific theoretical support. 


\section{References}

Fjell, A., Westlye, L., Amlien, I., Tamnes C.K., Grydeland, H., Engvig, A., Espeseth, T., Reinvang, I., Lundervold, A.J., \& Lundervold, A. (2015). High-expanding cortical regions in human development and evolution are related to higher intellectual abilities. Cerebral Cortex, 25(1), 26-34. https://dx.doi.org/10.1093/cercor/bht201

Foster, J., Lyte, M., Meyer, E., \& Cryan, J. (2016). Gut Microbiota and Brain Function: An Evolving Field in Neuroscience. International Journal of Neuropsychopharmacology, 19(5), 1-114. https://dx.doi.org/10.1093/ijnp/pyv114

Gu, S., Pasqualetti, F., Cieslak, M., Telesford, Q.K., \& Yu, A.B. (2015). Controllability of structural brain networks. Nature communications, 6, 8414. https://dx.doi.org/10.1038/ncomms9414

Herrmann, N. (1997). The Whole Brain Business Book. Oversea Publishing House.

Li, R.H., Dai, X., \& Chen, A. (2012). A study on the correlation between cognitive function and physical exercise of the elderly in Yangzhou. Course Education Research, 16, 10-11.

Li, W., \& Wang, Q. (2012). Effects of different sports on exercise induced emotion of college students. Journal of Shaanxi University of Science and Technology, 30(1), 174-177. https://dx.doi.org/10.3969/j.issn.10005811.2012.01.041

Saxena, G., \& Flora, S.J. (2012). Changes in brain biogenic amines and haem biosynthesis and their response to combined administration of succimers and Centella asiatica in lead poisoned rats. Journal of Pharmacy and Pharmacology, 58(4), 547-559. https:/dx./doi.org/10.1211/jpp.58.4.0015

Vaynman, S., Ying, Z., \& Gomez-Pinilla, F. (2004). Hippocampal BDNF mediates the efficacy of exercise on synaptic plasticity and cognition. European Journal of Neuroscience, 20(10), 2580-2590. https://dx.doi.org/10.1111/j.1460-9568.2004.03720.x

Wi-Young So (2017). The Effect of Taekwondo Coach-Athlete Interaction on Perceived Performance: The Moderating Effect by Two Taekwondo Styles, Gyeorugi and Poomsae, Journal of Men 's Health, 13(2), e34e39; DOI: 10.22374/1875-6859.13.2.5

Yang, Z., Chang, C., Xu, T., Jiang, L., Handwerker, D.A., Castellanos, F.X., Milham, M.P., Bandettini P.A., \& Zuo, X.N. (2014). Connectivity trajectory across lifespan differentiates the precuneus from the default network. NeuroImage, 89(1), 46-564. https:/dx./doi.org/10.1016/j.neuroimage.2013.10.039

Yang, Z., Zuo, X.N., Mc Mahon, K.L., Cameron Craddock, R., Kelly, C., de Zubicaray, G.I., Hickie, I., Bandettini, P.A., Xavier Castellanos, F., Milham, M.P., Wright, M.J. (2016). Genetic and environmental contributions to functional connectivity architecture of the human brain. Cerebral Cortex, 26(5), 2341-2352. https://dx.doi.org/10.1093/cercor/bhw027

Zhang, B. (2004). Thinking skills and physical education. Beijing: Beijing science and Technology Press. 